\title{
Intermediate outcomes of Ahmed glaucoma valve surgery in Asian patients with intractable glaucoma
}

\begin{abstract}
Objective To evaluate the efficacy and safety of Ahmed glaucoma valve (AGV) implantation in Asian patients with refractory glaucoma. Methods The study was a retrospective interventional case series conducted at a single institution between January 2004 and January 2006. The study population included 91 patients (91 eyes).

Results A total of 70 patients were successfully treated (74.5\%). Postoperatively, the median intraocular pressures declined significantly to $13 \mathrm{~mm} \mathrm{Hg}$ (interquartile range: $10-20 \mathrm{~mm} \mathrm{Hg}$ ) on day $1(P<0.001)$ and $17 \mathrm{~mm} \mathrm{Hg}$ (interquartile range: $12-19 \mathrm{~mm} \mathrm{Hg}$ ) at the last follow-up examination $(P<0.001)$. The cumulative probability of success according to KaplanMeier life-table analysis was $74 \%$ at 12 months and $43 \%$ at 2 years. Hazard of failure increased slightly with age, HR: 1.03 (95\% confidence interval $(\mathrm{CI})=1.00-1.05 ; P=0.044)$. The most common complication was hyphaemia at 12.77\%. There were no serious complications involving loss of visual acuity or sight. Conclusions AGV implantation is an acceptable treatment for refractory glaucoma in high-risk patients with few additional options. Eye (2010) 24, 547-552; doi:10.1038/eye.2009.181; published online 24 July 2009
\end{abstract}

Keywords: Ahmed glaucoma valve surgery; refractory glaucoma; AGV implantation; glaucoma drainage devices; glaucoma surgery

\section{Introduction}

Implantation of glaucoma drainage devices (GDDs) has assumed an important role in the surgical treatment of complicated and refractory

M-C Tai, J-H Cheng, J-T Chen, C-M Liang

and D-W Lu

glaucoma. These devices can be used as the primary surgical modality as well as a secondary procedure for those patients treated with earlier surgery, such as trabeculectomy with or without antimetabolite therapy. ${ }^{1,2}$ GDDs, including the Ahmed glaucoma valve (AGV), facilitate aqueous drainage through a tube inserted into the anterior or posterior chamber connected to a posterior plate sutured into the episclera. ${ }^{1}$ The devices may be valved or unvalved and assist passive diffusion of aqueous humour from the anterior chamber. The AGV has a $185-\mathrm{mm}^{2}$ polypropylene plate and a Venturi-type unidirectional valve consisting of a folded silicone elastomer membrane, with the free edge forming a one-way outlet designed to open at a pressure of $8 \mathrm{~mm} \mathrm{Hg}$. A valved GDD, such as the $\mathrm{AGV}$, minimizes the incidence of postoperative hypotony. ${ }^{3,4}$ Initial clinical experience found a reduced rate of postoperative hypotony with valved GDDs, ${ }^{4,5}$ but long-term studies on nonvalved GDDs indicate better final outcomes in reduced intraocular pressures (IOPs). ${ }^{6}$

Thus far, no discrepancies have been observed in outcomes for GDD implants in Asian versus non-Asian eyes, although Asians have been observed to have more severe tissue reactions. ${ }^{4,7}$ The purpose of this study was to evaluate the clinical experience of using AGV in Asian patients with intractable glaucoma. Differences in pre- and postoperative intraocular pressure, risks for failure, and surgical complications up to 2 years after surgery were investigated.

\section{Materials and methods}

We reviewed the medical records of patients treated with AGV implantation for intractable
Department of

Ophthalmology, National Defense Medical Center, Tri-Service General Hospital, Taipei, Taiwan, Republic of China

Correspondence: D-W Lu, Department of Ophthalmology, National Defense Medical Center, Tri-Service General Hospital, No. 325, Sec 2, Cheng Gong Road, Nei-Hu, Taipei 114, Taiwan, Republic of China

Tel: + 886287927165 ;

Fax: + 886287927164 .

E-mail: p310849@

ms23.hinet.net

Received: 10 June 2008 Accepted in revised form: 11 June 2009 Published online: 24 July 2009 
glaucoma uncontrolled by medications and laser/ surgical interventions at the Department of Ophthalmology, Tri-Service General Hospital, Taipei, Taiwan between January 2004 and January 2006 for this retrospective interventional case series. A total of 91 patients (91 eyes) were included from this single institution. All surgeries were performed by the corresponding author and no other GDDs were used during the study period. Exclusion criteria included the inability to be followed up for an extended period of time postoperatively. The study was approved by the hospital's institutional review board, and signed written consent was obtained from each patient postoperatively to allow use of the follow-up clinical record.

Pre- and postoperative documentation of patient demographics for statistical analysis included age, gender, detailed clinical history, results of slit-lamp examinations, IOP measurements using the Goldmann applanation tonometer (Haag-Streit, Bern, Switzerland), and best-refracted visual acuity (VA) measurement with a standard logMAR chart. The distance VA was measured according to the standard procedure developed for the Early Treatment of Diabetic Retinopathy Study. ${ }^{8}$ These measurements were performed at $4 \mathrm{~m}$.

Under peribulbar anaesthesia, we created a fornixbased conjunctival flap in the superotemporal quadrant between two adjacent recti muscles. The rectus muscles were identified, but no attempt was made to isolate them. After a $3 \times 3 \mathrm{~mm}$ triangular, scleral flap was created, the AGV (model S2 with a $185-\mathrm{mm}^{2}$ polypropylene plate, New World Medical, Rancho Cucamonga, CA, USA) was irrigated with balanced saline solution (BSS, Alcon, Fort Worth, TX, USA) to prime the valve mechanism. The polypropylene body was placed $8 \mathrm{~mm}$ posterior to the corneoscleral limbus and sutured to the sclera with an 8-0 prolene suture (Ethicon Inc., Somerville, NJ, USA). The tube was trimmed so that the bevel faced the corneal endothelial surface and then inserted into the an anterior chamber through a needle track made with a 23-gauge needle. A human donor patch graft (scleral or pericardial patch graft) (TranZgraft, Tissue Banks International, San Rafael, CA, USA) was placed on the tube with the anterior edge adjacent to the limbus and sutured to the sclera with an 8-0 prolene suture, then anterior chamber injection of $0.5 \mathrm{~cm}^{3}$ Viscoelastic (Healon GV, Advanced Medical Optics, Santa Ana, CA, USA) was performed to avoid early hypotony. The conjunctiva was sutured to the limbus, and all eyes received subconjunctival injections of steroids and antibiotics at the end of the procedure. No adjunctive metabolite was used.

Postoperatively, topical $0.3 \%$ ofloxacin (Tarvid, Santen, Osaka, Japan) and 1\% prednisolone acetate eye drops (Allergan, Irvine, CA, USA) were prescribed and tapered slowly over 4-8 weeks. Antiglaucoma medications were adjusted on the basis of the IOP and clinical status of the operated eye. Patients were examined at predetermined postoperative intervals: 1 day, 1 week, 2 weeks, 1 month, 3 months, 6 months, 9 months, 1 year, and 2 years. Slit-lamp examinations and assessments of VA, IOP, and complications were performed at each of these visits.

The primary outcome variable for success was postoperative IOP control. Absolute success was defined as an IOP $\leqslant 21 \mathrm{~mm} \mathrm{Hg}$ and $>6 \mathrm{~mm} \mathrm{Hg}$ without visually impairing complications, loss of light perception, or the need for additional glaucoma surgery or AGV implant removal. Hypotony was defined as IOP $<6 \mathrm{~mm} \mathrm{Hg}$ on any single visit. For IOP $\geqslant 21 \mathrm{~mm} \mathrm{Hg}$ or $\leqslant 6 \mathrm{~mm} \mathrm{Hg}$, treatment was attempted to raise or lower the IOP and the patient was re-examined within a week; therefore, patients may have received additional postoperative examinations. Success or failure was not defined until the patient had been seen at least twice after the 3-6-month time frame. The other outcome parameters were operative and postoperative complications, including hyphema, shallow anterior chamber, hypotony, choroidal effusion, graft failure, plate exposure with leak, scleral patch graft or tube exposure, and tube blockage.

To assess differences in IOPs and logMAR across multiple time points, we used Friedman's test. Moreover, differences between pre- and postoperative IOP and $\log$ MAR on day 1 , and IOPs and $\log$ MARs at each follow-up examination were analysed using Wilcoxon's signed-rank tests. Non-parametric data were presented as median (interquartile range), while categorical data were represented by number and percentage. KaplanMeier life-table analysis was used to calculate survival curves, and Cox's proportional hazards model used to determine univariate risk factors for failure. Rescaled Schoenfeld residuals were obtained. All statistical assessments were two-sided and were evaluated at the $P \leqslant 0.05$ level for significant difference. Statistical analyses were performed using SPSS 15.0 statistics software (SPSS Inc., Chicago, IL, USA).

\section{Results}

Demographic and preoperative diagnoses for the 91 subjects are indicated in Table 1 . Patients ranged in age from 40 to 66 years (median 55 years) and had undergone at least two earlier glaucoma-filtering surgeries or laser peripheral iridotomy or trabeculoplasty. The most common preoperative diagnoses were primary open angle $(41.8 \%)$, neovascular (24.2\%), and uveitis glaucoma $(14.3 \%)$. The majority of patients had pseudophakic $(71.4 \%)$ or phakic glaucoma (18.7\%). The median follow-up was 24 months. 
Figure 1 represents the IOP data during the postoperative follow-up period for all patients. According to Friedman's test, IOP differed significantly over time $(P<0.001)$. The median preoperative IOP was $30 \mathrm{~mm} \mathrm{Hg}$ (25th percentile $=26 \mathrm{~mm} \mathrm{Hg}$; 75th percentile $=41 \mathrm{~mm} \mathrm{Hg}$ ). Postoperatively, the median IOP declined significantly to $13 \mathrm{~mm} \mathrm{Hg}$ (interquartile range: $10-20 \mathrm{~mm} \mathrm{Hg})$ on day $1(P<0.001)$, rising slightly to

Table 1 Demographic and preoperative diagnoses $(n=91)$

\begin{tabular}{lc}
\hline & All $(\mathrm{n}=91)$ \\
\hline Age (years) & $55.03(40.00,66.02)$ \\
Gender (male) & $47(51.6 \%)$ \\
& \\
Preoperative diagnosis (glaucoma type) & \\
$\quad$ Uveitis & $13(14.3 \%)$ \\
Congenital & $9(9.9 \%)$ \\
Primary open angle & $38(41.8 \%)$ \\
Angle closure & $9(9.9 \%)$ \\
Neovascular & $22(24.2 \%)$ \\
& \\
Lens status & \\
Pseudophakia & $65(71.4 \%)$ \\
Aphakia & $9(9.9 \%)$ \\
Phakia & $17(18.7 \%)$ \\
Diabetes mellitus & $24(26.4 \%)$ \\
Preoperative IOP (mm Hg) & $30(26,41)$ \\
Preoperative logMAR & $0.7(0.4,1.0)$ \\
No. of earlier surgeries & $2(1,2)$ \\
Length of follow-up (months) & $24(12,36)$ \\
\hline
\end{tabular}

Abbreviations: No., number; IOP, intraocular pressure.
$17 \mathrm{~mm} \mathrm{Hg}$ (interquartile range: $12-19 \mathrm{~mm} \mathrm{Hg}$ ) at the last follow-up $(P<0.001)$. Moreover, the Wilcoxon signedrank tests showed a significant increase between 1 day and 1 month postoperatively $(P=0.035), 1$ day and 6 months $(P<0.001)$, and 1 day and 2 years $(P=0.017)$. Eight subjects had early postoperative IOP values exceeding $23 \mathrm{~mm} \mathrm{Hg}$. Of these, two were diagnosed with traumatic glaucoma, two with neovascular glaucoma, and the remaining four were diagnosed as follows: one each with Cogan-Reese syndrome, silicon oil glaucoma, aphakic glaucoma, and uveitic glaucoma. All eight had an encapsulated bleb during exploratory bleb tapping before iridotomy and AGV implantation.

The cumulative probability of success as per Kaplan-Meier life-table analysis was $74 \%$ at 12 months and $43 \%$ at 2 years (data not shown). The age, gender, diagnosis, lens status, history of DM, number of earlier operations and preoperative IOP were analysed as potential risk factors for failure in AGV implantation as shown in Table 2. Cox proportional hazards model showed a small increase in the hazard of failure with age, HR: 1.03 (95\% confidence interval $(\mathrm{CI})=1.00-1.05$; $P=0.044)$. Postoperative complications were as follows: hyphaemia $(n=12)$, shallow anterior chamber $(n=9)$, hypotony $(n=6)$, choroidal effusion $(n=5)$, and vitreous tube blockage $(n=4)$. These complications occured in the first 3 months after surgery as shown in Table 3. Late complications observed were scleral patch graft exposure $(n=3)$, plate exposure with wound leak $(n=2)$, and tube extrusion $(n=2)$. Patients were examined for VA at predetermined postoperative intervals. VA remained

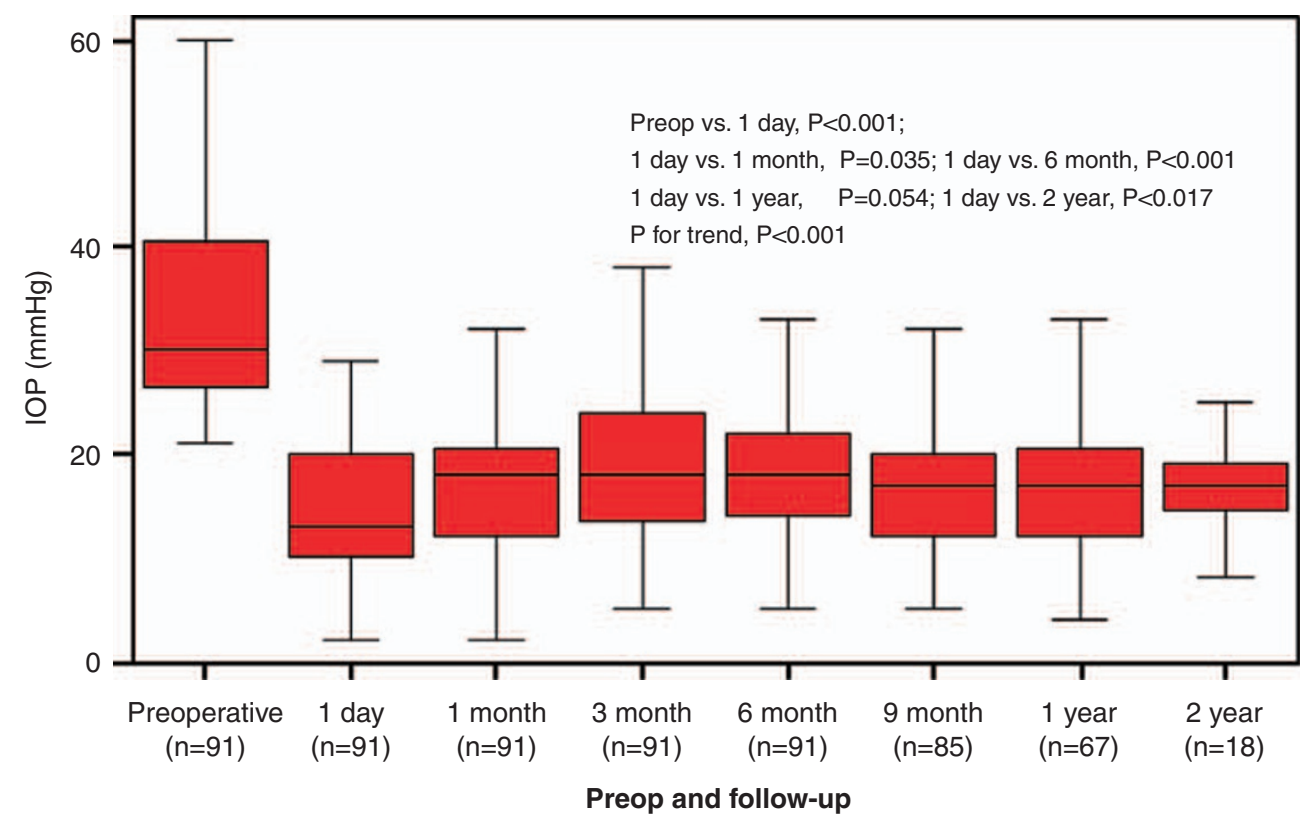

Figure 1 Pre- and postoperative intraocular pressures after Ahmed glaucoma valve implantation surgery plotted over time. The graph shows a marked decrease in median IOP from baseline. Note the hypertensive phase at 3 months after implantation. 
Table 2 Univariate analysis of risk factors for failure in AGV implants

\begin{tabular}{|c|c|c|c|}
\hline & Hazard Ratio & $95 \% \mathrm{CI}$ & P-value \\
\hline Age (years) & 1.03 & $(1.00,1.05)$ & $0.044^{\mathrm{a}}$ \\
\hline \multicolumn{4}{|l|}{ Gender } \\
\hline Male & 1.00 & - & \\
\hline Female & 1.38 & $(0.55,3.44)$ & 0.494 \\
\hline \multicolumn{4}{|l|}{ Diagnosis (glaucoma type) } \\
\hline Uveitis & 1.00 & - & \\
\hline Congenital & 0.55 & $(0.05,6.79)$ & 0.644 \\
\hline Primary open angle & 3.44 & $(0.64,18.41)$ & 0.149 \\
\hline Angle closure & 5.37 & $(0.67,43.26)$ & 0.114 \\
\hline Neovascular & 4.92 & $(0.87,27.83)$ & 0.071 \\
\hline \multicolumn{4}{|l|}{ Lens status } \\
\hline Pseudophakia & 1.00 & - & \\
\hline Aphakia & NA & NA & NA \\
\hline Phakia & 2.06 & $(0.73,5.78)$ & 0.171 \\
\hline \multicolumn{4}{|l|}{ Diabetes mellitus } \\
\hline Present & 1.00 & - & \\
\hline Absent & 1.28 & $(0.50,3.33)$ & 0.607 \\
\hline \multicolumn{4}{|l|}{ Earlier surgery } \\
\hline One & 1.00 & - & \\
\hline Two & 0.63 & $(0.22,1.83)$ & 0.395 \\
\hline Three & 0.62 & $(0.19,2.08)$ & 0.441 \\
\hline Preoperative IOP & 0.99 & $(0.95,1.04)$ & 0.757 \\
\hline
\end{tabular}

Abbreviations: CI, confidence interval; IOP, intraocular pressure.

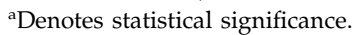

Table 3 Postoperative complications

\begin{tabular}{lcc}
\hline & $\begin{array}{c}\text { Early } \\
(0-3 \text { months })\end{array}$ & $\begin{array}{c}\text { Late } \\
(>3 \text { months })\end{array}$ \\
\hline Hyphema & $12(12.77 \%)$ & 0 \\
Shallow anterior chamber & $9(9.57 \%)$ & 0 \\
Hypotony & $6(6.38 \%)$ & 0 \\
Choroidal effusion & $5(5.32 \%)$ & 0 \\
Graft failure & $1(1.06 \%)$ & $1(1.06 \%)$ \\
Plate exposure with wound leak & $1(1.06 \%)$ & $1(1.06 \%)$ \\
Scleral patch graft exposure & $2(2.13 \%)$ & $3(3.19 \%)$ \\
Tube extrusion & $1(1.06 \%)$ & $2(2.13 \%)$ \\
Vitreous blocking tube & $4(4.26 \%)$ & $1(1.06 \%)$ \\
\hline
\end{tabular}

stable throughout the study period (median $=0.7$, $P=0.610$ ) (data not shown).

\section{Discussion}

Valved and non-valved GDDs have been used successfully to treat a variety of glaucomas that are resistant to other therapies. In our study, the median
IOP decreased to $13 \mathrm{~mm} \mathrm{Hg}$ at day 1 and $17 \mathrm{~mm} \mathrm{Hg}$ at 2 years. This is comparable to other studies showing a decreased IOP at day 1 of $10-13 \mathrm{~mm} \mathrm{Hg}$ and $13-17 \mathrm{~mm} \mathrm{Hg}$ at 1 year. ${ }^{1-4}$ We found a cumulative probability of success for AGV implantation of $74 \%$ at 12 months and $43 \%$ at 2 years. These values are in general lower than those published earlier. Huang ${ }^{2}$ reported a cumulative probabilty of success of $87 \%$ at 1 year and $75 \%$ at 2 years, Toupozis ${ }^{9}$ reported $87 \%$ at 1 years and $82 \%$ at 2 years and $\mathrm{Lai}^{7}$ showed a success rate of $73.8 \%$ in a study population of $98 \%$ Asian eyes and using similar criteria for success. However, in the long-term study of Topouzis et $a l^{9}$ on patients with AGV implantation found shorter lengths of follow-up among Asians and Hispanics and increased odds of failure in these groups.

The relatively low success rate in our study, $43 \%$ at 2 years, may be explained by the inclusion of eyes with poorer diagnoses. Most of our patients had pseudophakic primary open angle and neovascular glaucoma. In general, AGV has good prognosis in neovascular glaucoma patients, but this group included patients with diabetes. Neovascular glaucoma is a major ocular complication associated with diabetes mellitus. Therefore, such patients have a higher probability of hyphaemia and recurrent vitreous haemorrhage postoperation, which might result in failure owing to high pressure. In fact, we found neovascular glaucoma to be among those diagnoses with increased likelihood of failure (4.92), along with angle closure (5.37) and primary open angle (3.44) (Table 2). Huang reported a $68 \%$ success rate and Lai $46.5 \%$ success rate in patients with neovascular glaucoma. ${ }^{2,7}$ Wilson had a $69.8 \%$ success rate at 42 months with inclusion of corneal grafts, neovascular, and uveitic glaucoma. ${ }^{3,10}$

The advanced age in our study cohort may also have affected our success rate. Ayyala $e t a^{1}$ showed a cumulative probability of success of $77 \%$ at 1 year in a study population with an average age of 64 years. Huang's study population averaged 60.9 years $^{2}$ and Lai's study population averaged 49.9 years. ${ }^{7}$ Our study population averaged 55.03 years (range 40-66 years). In addition, we found a significantly increased risk for failure in elderly patients in our study cohort. Not only were these older subjects, but also, in general, they had at least two surgical glaucoma procedures before tube insertion, which is more than the current average. Cox proportional hazards model indicated that the likelihood of failure in AGV implants significantly increased with age (OR 1.03, 95\% CI $=1.00-1.05 ; P=0.044)$. We agree that prospective studies should include age as a risk factor, although its effect is weak. Furthermore, no publication has yet reported a prospective trial of the Ahmed valve with age as a risk factor. This knowledge 
gap may be due to the difficulty in recruiting samples at different ages to allow for randomization in a prospective study of this type of therapy.

However, a recent retrospective study reviewed patients who had Molteno implants inserted for neovascular glaucoma. The authors concluded that age, sex, and postoperative intraocular pressure control did not predict the survival. ${ }^{11}$ Another retrospective study for determining the intermediate-term efficacy and safety of Baerveldt glaucoma implants in Asian eyes with complicated glaucoma ${ }^{12}$ found that preoperative IOP, length of follow-up, increasing age (OR 1.05; 95\% CI $=1.01-1.09 ; P=0.02$ ), and number of operations performed before implant surgery (OR 1.57; 95\% CI =1.07-2.31; $P=0.01$ ) were correlated positively with failure after adjusting for gender. However, the role of age as a risk factor for AGV failure is rather weak, amounting to somewhere between 1 and $5 \%$.

The most common early postoperative complication in our study was hyphaemia (12.77\%), followed by shallow anterior chamber $(9.57 \%)$ and hypotony $(6.38 \%)$. Hyphema has been reported at 13.8-16.9\% in AGV implantations and typically resolves without surgical intervention. 6,10,11 The reported incidence range of shallow anterior chamber with hypotony is $3.5-27 \%$ for valved and non-valved GDDs, with the Ahmed valve at $3.5 \%$, Molteno at 5\%, and the Baerveldt at $16.5 \%{ }^{12}$ For hypotony alone, Taglia ${ }^{4}$ reported an incidence of $31 \%$ for AGV implantation, 77\% for Krupin, and 52\% for Molteno, in a study of 53 patients comparing these three devices. Differences between these reports and our finding might be explained by the variety in patients' clinical status and in surgical technique. In our study, anterior chamber injection of $0.5 \mathrm{~cm}^{3}$ Viscoelastic (Healon $\mathrm{GV}$ ) was performed to avoid early hypotony after suture. The incidence of choroidal effusion was $5.32 \%$, which is comparable with the $7-33 \%$ reported using different devices in varying series..$^{2,3,12,13}$ Postoperative choroidal effusion often occurs because of lack of resistance to aqueous outflow even in valve implants, especially after excessive straining. Fortunately, the condition typically resolves spontaneously, with infrequent need for surgical drainage. No serious complications occurred that involved loss of VA or sight. Graft failure and scleral patch graft exposure were minimal (5.4\%), probably because of the use of the frozen graft patch or lack of manipulation of the rectus muscles during AGV installation. Thus, the use of these grafts is economical and offers high tensile strength and excellent protection against tube erosion, a condition not seen in our series. ${ }^{10}$

In conclusion, $\mathrm{AGV}$ implantation seems to be a viable option for Asian patients with refractory glaucoma. In this series of 91 patients, no serious adverse events were observed and the device showed an overall cumulative probability of success $74 \%$ at 1 year and $43 \%$ at 2 years. AGV implantation seems to be an acceptable treatment for refractory glaucoma in high-risk patients with few additional options.

\section{Acknowledgements}

This study was supported by funding from the Tri Service General Hospital (TSGH-C97-1-SO4). We have no additional financial disclosures. M-C Tai, J-H Cheng, J-T Chen, and C-M Liang collected the data and reviewed the literature. D-W Lu was surgeon and chief editor. The study was approved by the institutional review board of Tri-Service General Hospital. All patients gave written informed consent for the surgical procedures. As the study was a review of records developed during the course of medical treatment, no informed consent was obtained for study inclusion. We thank Medcom Asia for providing statistical assistance.

\section{References}

1 Ayyala RS, Zurakowski D, Smith JA, Monshizadeh R, Netland PA, Richards DW et al A clinical study of the Ahmed glaucoma valve implant in advanced glaucoma. Ophthalmology 1998; 105: 1968-1976.

2 Huang MC, Netland PA, Coleman AL, Siegner SW, Moster MR, Hill RA. Intermediate-term clinical experience with the Ahmed glaucoma valve implant. Am J Ophthalmol 1999; 127: 27-33.

3 Wilson MR, Mendis U, Smith SD, Paliwal A. Ahmed glaucoma valve implant vs trabeculectomy in the surgical treatment of glaucoma: a randomized clinical trial. Am J Ophthalmol 2000; 130: 267-273.

4 Taglia DP, Perkins TW, Gangnon R, Heatley GA, Kaufman PL. Comparison of the Ahmed glaucoma valve, the Krupin eye valve with Disc, and the double-plate Molteno implant. J Glaucoma 2002; 11: 347-353.

5 Coleman AL, Hill R, Wilson MR, Choplin N, Kotas-Neumann $\mathrm{R}$, Tam $\mathrm{M}$ et al Initial clinical experience with the Ahmed glaucoma valve implant. Am J Ophthalmol 1995; 120: 23-31.

6 Ayyala RS, Zurakowski D, Monshizadeh R, Hong CH, Richards D, Layden WE et al. Comparison of double-plate Molteno and Ahmed glaucoma valve in patients with advanced uncontrolled glaucoma. Ophthalmic Surg Lasers 2002; 33: 94-101.

7 Lai JS, Poon AS, Chua JK, Tham CC, Leung AT, Lam DS. Efficacy and safety of the Ahmed glaucoma valve implant in Chinese eyes with complicated glaucoma. Br J Ophthalmol 2000; 84: 718-721.

8 Ferris 3rd FL, Kassoff A, Bresnick GH, Bailey I. New visual acuity charts for clinical research. Am J Ophthalmol 1982; 94: 91-96.

9 Topouzis F, Coleman AL, Choplin N. Follow-up of the original cohort with the Ahmed glaucoma valve implant. Am J Ophthalmol 1999; 128: 198-204. 
10 Wilson MR, Mendis U, Paliwal A, Haynatzka V. Long-term follow-up of primary glaucoma surgery with Ahmed glaucoma valve implant versus trabeculectomy. Am J Ophthalmol 2003; 136: 464-470.

11 Blanc JP, Molteno AC, Fuller JR, Bevin TH, Herbison P. Life expectancy of patients with neovascular glaucoma drained by Molteno implants. Clin Exp Ophthalmol 2004; 32: 360-363.
12 Seah SK, Gazzard G, Aung T. Intermediate-term outcome of Baerveldt glaucoma implants in Asian eyes. Ophthalmology 2003; 110: 888-894

13 Kook MS, Yoon J, Kim J, Lee MS. Clinical results of Ahmed glaucoma valve implantation in refractory glaucoma with adjunctive mitomycin C. Ophthalmic Surg Lasers 2000; 31: 100-106. 